\title{
Are renewables really that expensive? The impact of uncertainty on the cost of the energy transition
}

\author{
Xiang $\mathrm{Li}^{\mathrm{a}}{ }^{,}$, Stefano Moret ${ }^{\mathrm{a}}$, Francesco Baldi ${ }^{\mathrm{a}}$ and François Maréchal ${ }^{\mathrm{a}}$ \\ a Industrial Process and Energy Systems Engineering, Ecole Polytechnique Fédérale de Lausanne, \\ Sion 1951, Switzerland \\ *xiang.li@epfl.ch
}

\begin{abstract}
The dramatic evidence of climate change is making the transition to more renewable energy systems an urgent global priority. As energy planners normally look 20-50 years ahead, it is crucial to consider the key uncertainties stemming from inaccurate forecasts (e.g. of fuel prices, investment costs, etc.) in energy models to ensure making robust investment decisions. Nonetheless, uncertainty is to date seldom accounted for in energy planning models.

In this paper, we challenge the general perception that the transition to a more renewable energy system always comes at a higher price; to do this, we analyze the impact of uncertainty on the cost of this transition for a real-world national energy system. Concretely, we first generate a set of energy planning scenarios with increasing renewable energy penetration (REP); in a second stage, we perform an uncertainty analysis to compare the cost of these scenarios and thus to determine the significance of the difference in their total cost in presence of uncertainty. Our results show that increasing the amount of renewable energy in a national energy system is not necessarily associated to a higher cost, and can even lead to a cost reduction for some specific realizations of the uncertain parameters.
\end{abstract}

Keywords: Energy planning, Uncertainty, Energy transition, Mixed-integer linear programming

\section{Introduction}

The Earth is warming up at an alarming pace. Most countries have agreed on ambitious goals for reducing the human impact on the climate (Rogelj et al., 2016) and have started to make plans for a transition towards more sustainable systems (Kern and Rogge, 2016). A major part of this energy transition is related to an increased use of renewable energy sources (RESs). Many authors have thus used energy models to evaluate the impact of an increased share of RESs in energy systems planning. Jacobson et al. (2015) investigated the feasibility and cost of a fully renewablepowered system for the United States and concluded that, when externalities are accounted for, such system would be less expensive than a traditional energy system; Schill (2014) performed a similar analysis for the German energy system with the objective of achieving $86 \%$ REP by 2050 , showing that this can be done with a relatively limited investment in energy storage capacity as long as a higher flexibility in the operation of fossil power plants is allowed.

Despite these results, and despite the fact that today there are several technological options for a cost-competitive generation of renewable energy, the public opinion often still perceives RESs as being far more expensive than fossil fuels. As an example, Ntanos et al. (2018) report the results of a recent survey in Greece, in which the respondents identified in the "high installation costs" the main reason for not using RESs. In this paper, we challenge this general perception. The main 


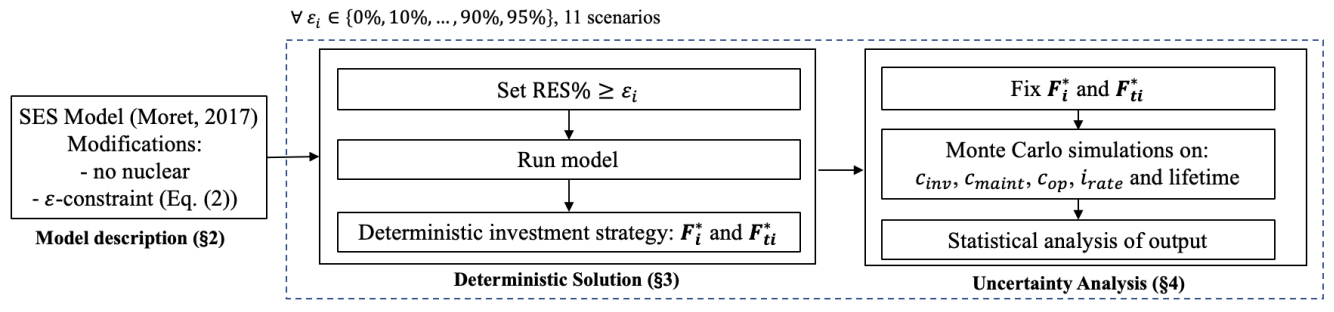

Figure 1: Modeling flowchart

contribution of our work compared to the literature is the consideration of multiple sources of uncertainty in the analysis. In fact, the main shortcoming of most works proposed in the literature lies in the use of "deterministic" models, which do not consider uncertainty and assume that longterm forecasts for the key parameters (e.g. fuel prices) are correct. However, long-term forecasts are inevitably inaccurate (Moret et al., 2017), and thus it is crucial to consider uncertainties in long-term energy modeling (DeCarolis et al., 2017; Mavromatidis et al., 2018).

In this paper, we propose an analysis of the cost and investment decisions for different degrees of REP in an energy system, with a particular focus on the impact of uncertainty in the process, taking the national energy system of Switzerland as a real-world case study. The methodological approach is shown in Figure 1. First, we model the Swiss energy system using the Swiss EnergyScope (SES) framework (Moret et al., 2014; Codina Gironès et al., 2015). SES, which we describe in Section 2, is a mixed-integer linear programming (MILP) model, which identifies the optimal investment and operation strategy to minimize the total annual cost of the energy system. In a second phase, the model is used deterministically (i.e. with all parameters at nominal values) to determine the optimal energy planning scenarios under increasing shares $\left(\varepsilon_{i}\right)$ of RESs (Section 3 ). Third, the impact of uncertainty is evaluated: for each of the previously obtained scenarios, the investment strategy is fixed and the total cost of the system is evaluated with a Monte-Carlo based approach, considering as uncertain parameters all the costs, the discount rates and the lifetime of technologies. This allows to perform an uncertainty analysis of the solution (Section 4).

\section{The energy model}

The paper is based on the SES model, a framework for the strategic energy planning of the Swiss energy system. In particular, in this paper we use the open-source MILP version of the model by Moret $(2017)^{1}$. It is a representative model of an energy system, including electricity, heating and mobility: given the end-use energy demand, the efficiency and cost of energy conversion technologies, the availability and cost of energy resources, the model identifies the optimal investment $(\mathbf{F})$ and operation $\left(\mathbf{F}_{\mathbf{t}}\right)$ strategies to meet the demand and minimize the total annual cost of the energy system. In comparison to other energy models, which often consider hourly timesteps and multistage investment plans, it has a lower level of detail, but it offers a reasonable trade-off between CPU time and accuracy; in particular, its multiperiod monthly formulation allows accounting for the main dynamics of concern in energy systems planning, such as seasonal variations, long-term energy storage management, and uncertainties in loads and renewable production.

The objective of the SES model is to minimize the annual total cost $\mathbf{C}_{\text {tot }}$ expressed by the sum of the annualized investment cost $\left(\mathbf{C}_{\mathbf{i n v}}\right)$, of the annual operational costs $\left(\mathbf{C}_{\mathbf{o p}}\right)$ and of the annual maintenance cost $\left(\mathbf{C}_{\text {maint }}\right)(1)$.

$$
\min \mathbf{C}_{\text {tot }}=\min \left(\sum_{j \in \mathscr{E}} \mathbf{C}_{\mathbf{i n v}}(j)+\sum_{j \in \mathscr{E}} \mathbf{C}_{\text {maint }}(j)+\sum_{r \in \mathscr{R}} \sum_{t \in \mathscr{T}} \mathbf{C}_{\mathbf{o p}}(r, t) t_{o p}(t)\right)
$$

\footnotetext{
${ }^{1}$ Model available at https://github.com/stefanomoret/SES_MILP and fully documented in (Moret, 2017)
} 
Are renewables really that expensive? The impact of uncertainty on the cost of the energy transition

where the sets $\mathscr{E}, \mathscr{R}$ and $\mathscr{T}$ represent the technologies, the resources (renewables and nonrenewables as well as electricity import) and the time periods (months) respectively. $t_{o p}(t)$ denotes the duration of the period $t$. The main constraints are the energy and resource balance in each period; the limited availability of resources; the capacity factor of different energy conversion technologies; the limits to the maximum installed capacity of different technologies; and the limits to grid capacity (accounting for the additional investments linked to a higher penetration of stochastic renewables).

In this paper, we modify the model presented by Moret (2017) as follows: $i$ ) we allow individual technologies to cover the full share of the demand in their sector, e.g. electric vehicles can satisfy all the private mobility demand $\left(f_{\min , \%}(j)=0, f_{\max } \%(j)=1 \forall j \in \mathscr{E}\right)$; ii) we allow freight transport to be fully satisfied by trains; iii) we impose the phase out of nuclear energy, in agreement with the Swiss energy strategy.

\section{Deterministic energy planning}

In addition, in order to quantify and to constrain the impact of RESs, Eq. (2) is added to the model formulation.

$$
\sum_{r \in \mathscr{R}_{R E S}} \sum_{t \in \mathscr{T}} \mathbf{F}_{\mathbf{t}}(r, t) t_{o p}(t) \geqslant \varepsilon \sum_{r \in \mathscr{R}} \sum_{t \in \mathscr{T}} \mathbf{F}_{\mathbf{t}}(r, t) t_{o p}(t)
$$

where the parameter $\varepsilon$ represents the minimal renewable penetration ratio in the energy system and the set $\mathscr{R}_{R E S}$ is a subset of $\mathscr{R}$ composed of all renewable resources (solar, wind, wood, hydro, geothermal, synthetic natural gas, bioethanol and biodiesel). In Eq. (2), $\mathbf{F}_{\mathbf{t}}(r, t)$ stands for the utilization of resource $r$ at period $t$. In this paper we analyze 11 scenarios with different values of the $\varepsilon$ parameter, ranging from $0 \%$ to $90 \%$ with $10 \%$ steps, plus the "maximum REP" scenario with $\varepsilon=95 \%$.

The energy mixes of the resulting 11 scenarios are shown in Figure 2. It should be noted that scenarios 1, 2 and 3 are equivalent, due to the fact that the REP in the unconstrained (i.e. with $0 \%$ minimum REP) case is approximately $20 \%$. Among the RESs, wind and (new) hydro-power are the most competitive from a cost perspective and reach their maximum potential at a REP level of

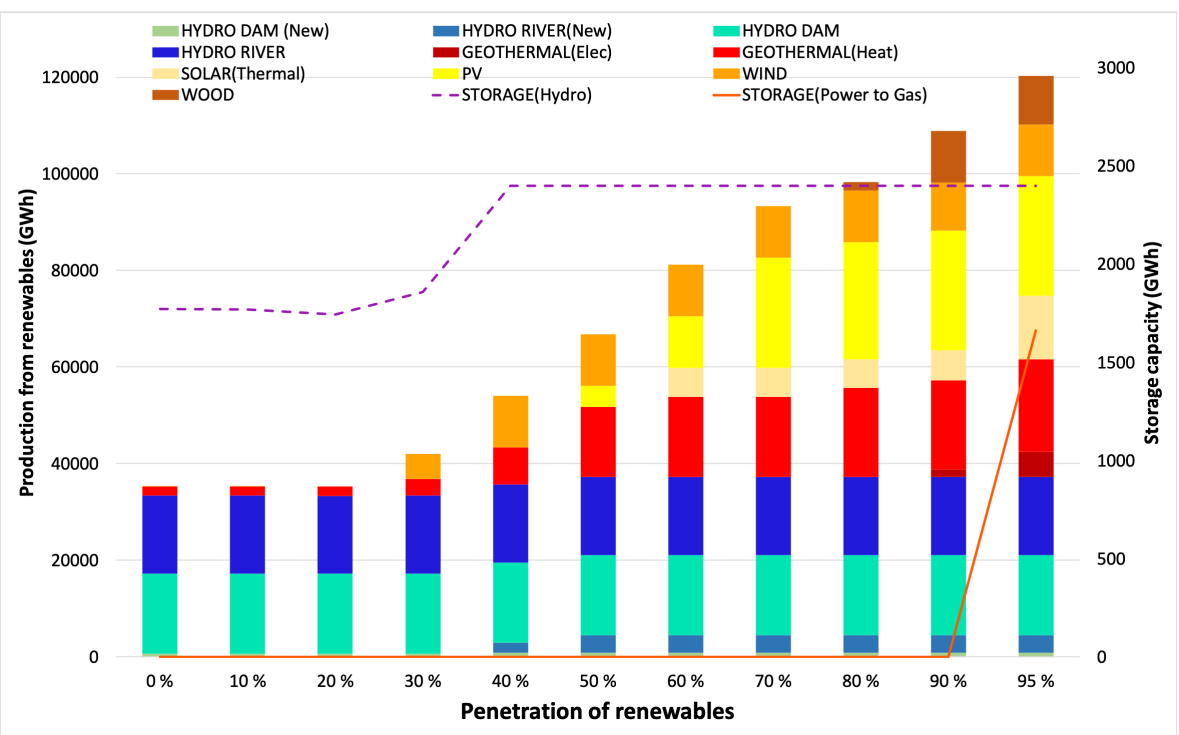

Figure 2: Renewable energy production and storage capacity in different REPs 


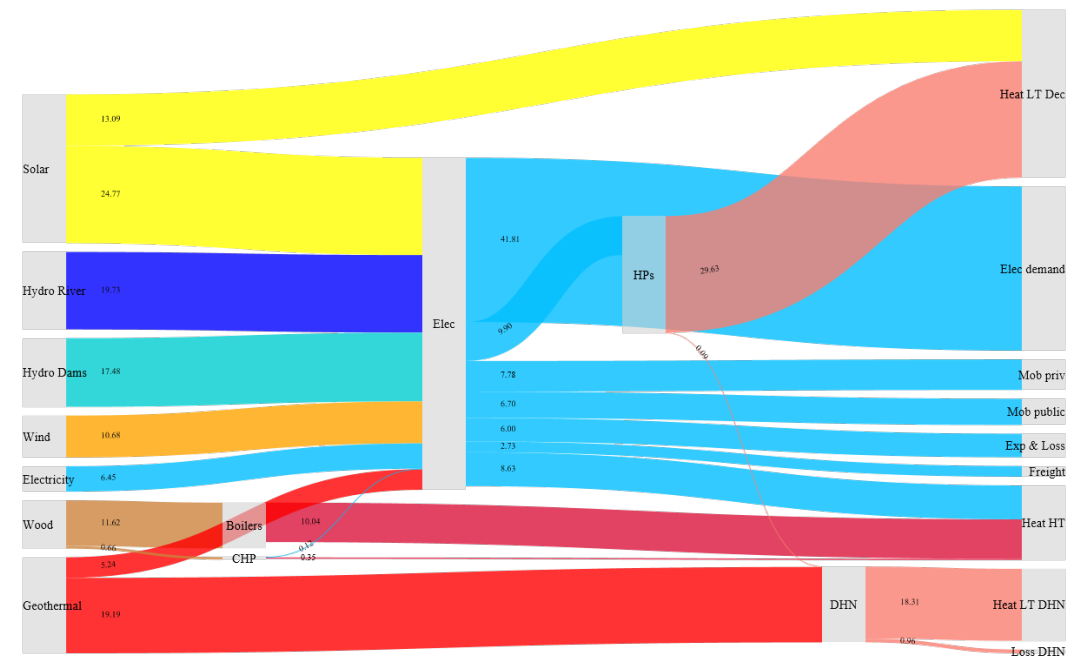

Figure 3: Sankey diagram for energy flows in $95 \%$ REP scenario [TWh]

$60 \%$. The use of solar energy emerges in the optimal solution only starting from a REP of $50 \%$, and it is only used extensively for REP $\geq 70 \%$. Wood is only used in very high REP scenarios, due to its high assumed cost in the model. The results of the deterministic optimization also show that the required storage capacity is relatively limited: hydro-electric storage reaches its peak in the $40 \%$ REP scenario, at a value approximately $35 \%$ higher than the base case, while power-to-gas is only activated in the $95 \%$ REP scenario, allowing to reach a total seasonal storage capacity of approximately 4,000 GWh. Note that, due to the monthly resolution of the model, here dynamic storage technologies for balancing in-time supply-demand at a daily or more fine time resolution (such as batteries) are not considered. This choice is motivated by computational reasons.

How does an almost completely renewable-based energy system look like? This is shown in Figure 3, where the energy flows in Scenario 11 are represented. As expected, the demand is dominated by the use of electricity, with the exception of part of the high-temperature heat demand (provided by biomass boilers) and of the low-temperature heat demand (provided by thermal solar and by geothermal heat). The use of boilers and gas-driven heat pumps for low-temperature heat is completely discontinued, substituted by electric heat pumps.

\section{Uncertainty analysis}

As highlighted in the Introduction, the problem of strategic energy planning is subject to a high degree of uncertainty, which should not be neglected. In this article, we consider five main uncertainty categories: interest rate, investment cost, maintenance cost, resource prices, and technology lifetime. In the SES model, this translates to a total of 157 uncertain parameters. We assume that all these uncertain parameters are uniformly distributed between their lower and upper bounds, adopting the methodology and the uncertainty ranges defined by Moret et al. (2017).

In the uncertainty analysis, the decision variables related to the installed capacity of each technology, and the energy utilization for each mobility technology in each period are fixed to the optimal values calculated in the deterministic optimization for each scenario $\left(\mathbf{F}^{*}\right.$ and $\mathbf{F}_{\mathbf{t}}{ }^{*}$, respectively). Then, for each of the 11 scenarios, the SES model is run for different realizations of the uncertain parameters with a Monte-Carlo approach. This corresponds to a situation where a decision for the strategic energy planning has been taken, and we aim to analyze the cost of the application of that specific strategy with different values for the uncertain parameters, corresponding to different 


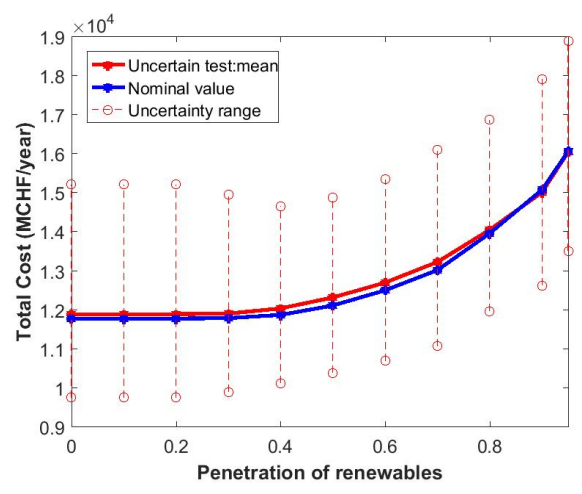

Figure 4: Nominal and uncertain mean total cost

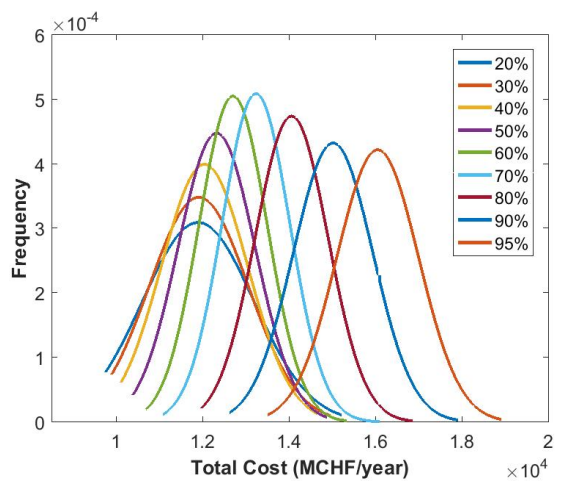

Figure 5: Nomalized total cost distribution

possible future scenarios. A total of 3,000 Monte-Carlo simulations (based on an observed convergence after 2,600 simulations) is run for each scenario.

In the Swiss energy system, a large share of the total annual cost (equal to 6,654 MCHF/y) derives from fixed costs, i.e. costs which are equal for all the solutions (such as the investment cost of existing hydroelectric power plants, of the electricity grid and of energy efficiency measures). In the simulation runs, we fix these costs to zero; this means that we do not consider them as uncertain in the simulations, but we add them to the simulation results at their nominal values.

Figure 4 shows the results of the uncertainty analysis. It can be osberved that the mean total cost values obtained by Monte-Carlo simulations are consistent with the deterministic results for each scenario, as in both cases the total cost increases with $\varepsilon$. However, the results also show that there is a high uncertainty in the total cost of the system for all REP levels, leading to a relatively high overlap between the different distributions (Figure 5). This can lead to the conclusion that, while on average increasing the share of renewable energy beyond $20 \%$ involves a higher cost for the system, this is not necessarily true once uncertainty is accounted for.

A higher REP has also an impact on the variance of the total cost (Figure 6): given the high uncertainty on the price of different fossil fuels, excluding them from the energy mix generates a clear reduction in the uncertainty of operational costs. In contrast, the uncertainty on the investment increases, partially balancing the previous effect, as a consequence of both the larger uncertainty of the investment cost of technologies activated with higher REP, and of the overall higher investment cost of the system, which amplifies the uncertainty related to the interest rate. Overall, it can be concluded that cost uncertainty decreases when the share of RES increases.

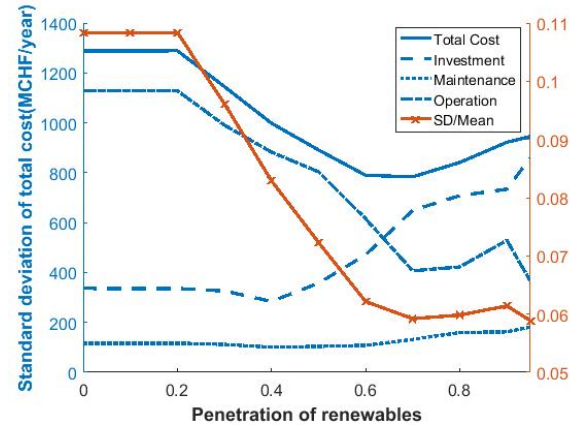

Figure 6: Total cost standard deviation breakdown

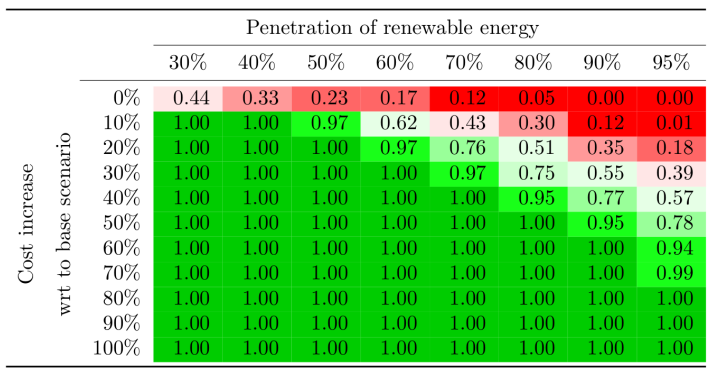

Figure 7: Probability of total cost increase for different REP scenarios 
Figure 7 shows the percentage of Monte-Carlo simulations where a given REP scenario was more expensive than the base case by a certain amount. An energy system with $70 \%$ REP was less than $20 \%$ more expensive than the base case in $76 \%$ of the simulations, and in $12 \%$ of the simulations it was actually less expensive than the base case. Even the $95 \%$ REP case was, in most (78 \%) cases, no more than $50 \%$ more expensive than the baseline case, while the less ambitious $50 \%$ REP scenario is at most $10 \%$ more expensive than the baseline. These results are in accordance with what previously published in the academic literature (e.g. Jacobson et al. (2015)), and the cost difference is expected to be even lower if the external costs were included in the analysis.

\section{Conclusions}

Are renewable really that expensive? Based on the work that we present in this paper, the answer is that yes, a renewable-based energy system will most likely be more expensive than one where we apply no constraint on the share of renewable energy, but not always, and not by much. Furthermore, a renewable-based energy system will be, overall, inherently more robust against unexpected developments, particularly with respect to fluctuations in fuel prices.

The results of our uncertainty analysis, in fact, reveal that the variance in the total cost of the system is high, and of the same order of magnitude of the average cost difference between different scenarios. This suggests that, when considering uncertainty, increasing the amount of renewable energy in a national energy system is not necessarily associated to a higher cost, and can even lead to a cost reduction in some specific realizations of the uncertain parameters. These results are of particular value to energy planners, as they challenge the common perception of a very high cost related to the energy transition towards a renewable energy-based system. While these conclusions are drawn based on a specific case study (the Swiss energy system), we consider that they are sufficiently general to be applicable to other countries.

\section{References}

V. Codina Gironès, S. Moret, F. Maréchal, D. Favrat, Oct. 2015. Strategic energy planning for large-scale energy systems: A modelling framework to aid decision-making. Energy 90, Part 1, 173-186.

J. DeCarolis, H. Daly, P. Dodds, I. Keppo, F. Li, W. McDowall, S. Pye, N. Strachan, E. Trutnevyte, W. Usher, M. Winning, S. Yeh, M. Zeyringer, May 2017. Formalizing best practice for energy system optimization modelling. Applied Energy 194, 184-198.

M. Z. Jacobson, M. A. Delucchi, G. Bazouin, Z. A. Bauer, C. C. Heavey, E. Fisher, S. B. Morris, D. J. Piekutowski, T. A. Vencill, T. W. Yeskoo, 2015. 100\% clean and renewable wind, water, and sunlight (wws) all-sector energy roadmaps for the 50 united states. Energy \& Environmental Science 8 (7), 2093-2117.

F. Kern, K. S. Rogge, Dec. 2016. The pace of governed energy transitions: Agency, international dynamics and the global Paris agreement accelerating decarbonisation processes? Energy Research \& Social Science 22, 13-17.

G. Mavromatidis, K. Orehounig, J. Carmeliet, Mar. 2018. Uncertainty and global sensitivity analysis for the optimal design of distributed energy systems. Applied Energy 214, 219-238.

S. Moret, 2017. Strategic energy planning under uncertainty. Ph.D. thesis, École Polytechnique Fédérale de Lausanne, Lausanne, Switzerland.

S. Moret, V. Codina Gironès, M. Bierlaire, F. Maréchal, Sep. 2017. Characterization of input uncertainties in strategic energy planning models. Applied Energy 202, 597-617.

S. Moret, V. Codina Gironès, F. Maréchal, D. Favrat, 2014. Swiss-EnergyScope.ch: A Platform to Widely Spread Energy Literacy and Aid Decision-Making. Chemical Engineering Transactions 39, 877-882.

S. Ntanos, G. Kyriakopoulos, M. Chalikias, G. Arabatzis, M. Skordoulis, Mar. 2018. Public Perceptions and Willingness to Pay for Renewable Energy: A Case Study from Greece. Sustainability 10 (3), 687.

J. Rogelj, M. den Elzen, N. Höhne, T. Fransen, H. Fekete, H. Winkler, R. Schaeffer, F. Sha, K. Riahi, M. Meinshausen, Jun. 2016. Paris Agreement climate proposals need a boost to keep warming well below $2{ }^{\circ} \mathrm{C}$. Nature 534 (7609), 631-639.

W.-P. Schill, Oct. 2014. Residual load, renewable surplus generation and storage requirements in Germany. Energy Policy 73, 65-79. 